\title{
MAJORIZATION AND DOMINATION IN THE BERGMAN SPACE
}

\author{
BORIS KORENBLUM AND KENDALL RICHARDS
}

(Communicated by Theodore W. Gamelin)

Abstract. Let $f$ and $g$ be functions analytic on the unit disk and let $\|\cdot\|$ denote the Bergman norm. Conditions are identified under which there exists an absolute constant $c$, with $0<c<1$, such that the relationship $|g(z)| \leq|f(z)|$ $(c \leq|z|<1)$ will imply $\|g\| \leq\|f\|$.

\section{INTRODUCTION}

Let $\mathbb{C}$ denote the complex plane, $\mathbb{D}$ the open unit disk, and $L^{2}(\mathbb{D})$ the Hilbert space of all measurable functions $f: \mathbb{D} \rightarrow \mathbb{C}$ with

$$
\|f\|^{2}=\frac{1}{\pi} \int_{\mathbb{D}}|f|^{2} d m<\infty,
$$

where $d m$ denotes the Lebesgue area measure. The Bergman space $A_{2}$ is defined to be the subspace of $L^{2}(\mathbb{D})$ consisting of functions analytic on $\mathbb{D}$.

Let $f$ and $g$ be analytic on $\mathbb{D}$. We say $g$ is majorized by $f$ on a region $R \subseteq \mathbb{D}$ if $|g(z)| \leq|f(z)|$ for all $z \in R$. By the positivity of $d m$, if $g$ is majorized by $f$ on $\mathbb{D}$ then certainly

$$
\|g\| \leq\|f\| \text {. }
$$

What other cases of majorization will imply (1)? In particular, when does majorization on an annulus imply (1)? That is, we investigate the existence of an absolute constant $c$, with $0<c<1$, such that if

$$
|g(z)| \leq|f(z)| \quad(c \leq|z|<1)
$$

then

$$
\|g\| \leq\|f\|
$$

In the case that either function is a monomial $z^{n}$, it has been shown (see [3]) that (2) implies (3) for any $c \leq 1 / \sqrt{3}$.

For arbitrary $f$ and $g$ analytic on $\mathbb{D}$, let $Z_{f}$ and $Z_{g}$ denote the zero sets (counting multiplicity) of $f$ and $g$, respectively. If $Z_{f} \backslash Z_{g}$ is empty, then (2) implies (3) for any $c \in(0,1)$, by the classical maximum principle.

Received by the editors May 16, 1991. Presented at AMS Special Session on Bergman Spaces in Baltimore, Maryland, on January 10, 1992.

1991 Mathematics Subject Classification. Primary 30C80, 30H05. 
Furthermore, it is known (see [1]) that when $Z_{g} \backslash Z_{f}$ is empty (2) will again imply (3) for $c \leq 1 /\left(2 e^{2}\right)$.

We seek conditions under which majorization on an annulus of inner radius $c \in(0,1)$ will imply $(3)$, without requiring either relative complementary zero set to be empty. We will show that such a $c$ exists when $Z_{g} \backslash Z_{f}$ and $Z_{f} \backslash Z_{g}$ are separated by an annulus.

\section{Preliminary Results}

First we present some definitions and preliminary facts that will be appealed to throughout this article (see [1,2]). Let $H^{\infty}$ be the space of all bounded analytic functions on $\mathbb{D}$, with

$$
\|h\|_{\infty}=\sup \{|h(z)| \mid z \in \mathbb{D}\} .
$$

For $G, F \in L^{2}(\mathbb{D})$, we say that $G$ is dominated by $F$ if $\|G h\| \leq\|F h\|$ for all $h \in H^{\infty}$, and we write $G \prec F$. It follows that if $G \prec F$ and $G, F \in$ $L^{\infty}(\mathbb{D})$, then $\|G h\| \leq\|F h\|$ for all $h \in A_{2}$. The following properties are direct consequences of the definition of domination.

Property 1. If $G, F \in L^{2}(\mathbb{D})$ and $G \prec F$, then $G \circ \phi \prec F \circ \phi$ for all Möbius transformations $\phi$ on $\mathbb{D}$.

Property 2. If $G_{i}, F_{i} \in H^{\infty}$ and $G_{i} \prec F_{i}$ for $i=1, \ldots, n$, then

$$
\left(G_{1} G_{2}, \ldots, G_{n}\right) \prec\left(F_{1} F_{2}, \ldots, F_{n}\right) .
$$

We denote $B_{a}(z)=\bar{a}(a-z) /[|a|(1-\bar{a} z)]$ for $a \in \mathbb{D}, a \neq 0$, and $B_{0}(z)=-z$.

Proposition 1. Let $a \in \mathbb{D}$ and $\gamma>0$. Then

$$
\left|B_{a}\right|^{\gamma((1+|a|) /(1-|a|))} \prec|z|^{\gamma},
$$

and

$$
\exp \{-2 \gamma(1+z) /(1-z)\} \prec|z|^{\gamma} .
$$

Corollary 1. Let $a \in \mathbb{D}$ and define $\alpha=(1-|a|) /(1+|a|), \beta=(1+|a|) /(1-|a|)$. Then $|z|^{\beta} \prec B_{a} \prec|z|^{\alpha}$.

(Proposition 1 is proved in [2] while Corollary 1 follows from (4) and Property 1.$)$

Corollary 2. Let $B(z)=\prod_{k=1}^{N} B_{a_{k}}(z),\left|a_{k}\right|<1$, and let

$$
\beta=\sum_{k=1}^{N}\left(\left[\frac{1+\left|a_{k}\right|}{1-\left|a_{k}\right|}\right]+1\right),
$$

where $N<\infty$ and $[\cdot]$ denotes the greatest integer function. Then $|z|^{\beta} \prec B$.

Proof. By Corollary 1, we have for each $k$ that $|z|^{\beta_{k}} \prec B_{a_{k}}$ where $\beta_{k}=$ $\left(1+\left|a_{k}\right|\right) /\left(1-\left|a_{k}\right|\right)$. Since $\beta_{k} \leq\left(\left[\beta_{k}\right]+1\right)$, we have

$$
z^{\left(\left[\beta_{k}\right]+1\right)} \prec B_{a_{k}} .
$$

Since both sides of (6) are in $H^{\infty}$, we can apply Property 2 for $k=1, \ldots, N$, which yields $|z|^{\beta} \prec B$. This proves the corollary. 
Proposition 2 (see [1]). Suppose $G \in H^{\infty},\|G\|_{\infty} \leq 1$, and $G(z) \neq 0$ for all $z \in \mathbb{D}$. If $|G(0)| \leq e^{-2 \gamma}$ for $\gamma>0$, then $G \prec|z|^{\gamma}$.

Proof. We have

$$
G(z)=\lambda \exp \left\{-\int_{\partial \mathbb{D}} \frac{\zeta+z}{\zeta-z} d \mu(\zeta)\right\},
$$

where $d \mu$ is a positive Borel measure on $\partial \mathbb{D}$, with $\mu(\partial \mathbb{D})=-\log |G(0)| \geq 2 \gamma$, and $|\lambda|=1$. Let $\eta=\mu(\partial \mathbb{D})$. Using the generalized arithmetic-geometric mean inequality combined with the Fubini Theorem and (5), we obtain for all $h \in A_{2}$

$$
\begin{aligned}
\int_{\mathbb{D}}|G h|^{2} d m & =\int_{\mathbb{D}} \exp \left\{-2 \eta \int_{0}^{2 \pi} \operatorname{Re} \frac{e^{i t}+z}{e^{i t}-z} \cdot \frac{d \mu(t)}{\eta}\right\}|h(z)|^{2} d m_{z} \\
& \leq \int_{\mathbb{D}}\left[\int_{0}^{2 \pi}\left|\exp \left\{\frac{-2 \eta\left(e^{i t}+z\right)}{e^{i t}-z}\right\}\right| \frac{d \mu(t)}{\eta}\right]|h(z)|^{2} d m_{z} \\
& =\int_{0}^{2 \pi} \int_{\mathbb{D}}\left|\exp \left\{\frac{-\eta\left(e^{i t}+z\right)}{e^{i t}-z}\right\} h(z)\right|^{2} d m_{z} \frac{d \mu(t)}{\eta} \\
& \leq \int_{\mathbb{D}}|z|^{\eta}|h(z)|^{2} d m \leq \int_{\mathbb{D}}|z|^{2 \gamma}|h(z)|^{2} d m .
\end{aligned}
$$

\section{MAIN RESUltS}

Theorem 1. Let $G \in H^{\infty}$ be such that $\|G\|_{\infty} \leq 1$ and $G(z) \neq 0$ for all $|z|<c$ for some $c \in(0,1)$. If $|G(0)| \leq\left(c^{(1+c) /(1-c)}\right)^{\gamma}$ for some $\gamma>0$, then $G \prec|z|^{\gamma}$.

(It is interesting to note that Proposition 2 is the limiting case of Theorem 1 since $c^{(1+c) /(1-c)} \rightarrow e^{-2}$ as $c \rightarrow 1^{-}$.) We postpone the proof of Theorem 1 until the end of the article. Once this theorem is proved, we can obtain the following results.

Theorem 2. Let $B(z)=\prod_{k=1}^{N} B_{a_{k}}(z)$, where $\left|a_{k}\right| \leq c<\frac{1}{3}$. Suppose $G \in H^{\infty}$, $\|G\|_{\infty} \leq 1$, and $G(z) \neq 0$ for all $|z|<d$, with $d>c$. If

$$
|G(0)| \leq\left(d^{(1+d) /(1-d)}\right)^{2 N}
$$

then $G \prec B$.

Proof. We have that $G \prec|z|^{2 N}$, by Theorem 1. Since $\left|a_{k}\right|<\frac{1}{3}$, it follows that

$$
\beta=\sum_{k=1}^{N}\left(\left[\frac{1+\left|a_{k}\right|}{1-\left|a_{k}\right|}\right]+1\right)=2 N .
$$

Thus, $|z|^{2 N} \prec B$, by Corollary 2. Therefore, $G \prec|z|^{2 N} \prec B$, which proves the theorem.

Theorem 3. Let $f, g \in A_{2}$. There exists an absolute constant $c_{0}$, with $0<$ $c_{0}<1$, such that for any $c<c_{0}$, if

(i) $|g(z)| \leq|f(z)| \quad(c \leq|z|<1)$ and

(ii) $Z_{g} \backslash Z_{f} \subset\left\{z \in \mathbb{D}\left|c^{1 / 3}<\right| z \mid<1\right\}$,

then $g \prec f$. 
Proof. Let $B$ be the finite Blaschke product

$$
B=\prod_{k=1}^{N} B_{a_{k}}, \quad \text { where }\left\{a_{k}\right\}_{k=1}^{N}=Z_{f} \backslash Z_{g} .
$$

Note that (i) implies $\left|a_{k}\right|<c$ for $k=1, \ldots, N$. Consider the function $G=g B / f$. It follows that $G \in H^{\infty},\|G\|_{\infty} \leq 1$, and $G(z) \neq 0$ for $|z|<c^{1 / 3}$. Also, on $|z|=c$, we have

$$
|G(z)| \leq|B(z)| \leq(2 c)^{N} .
$$

The classical maximal principle implies that $|G(0)| \leq(2 c)^{N}$. Letting $d=c^{1 / 3}$, it follows that $2 c \leq d^{2(1+d) /(1-d)}$ for $c<0.0021=c_{0}$. Thus,

$$
|G(0)| \leq\left(d^{(1+d) /(1-d)}\right)^{2 N}
$$

and $G(z) \neq 0$ for all $|z|<d$. Applying Theorem 2, we have $G \prec B$ or $\|G h\| \leq\|B h\|$ for all $h \in A_{2}$. Taking $h=h_{1} f / B$, with $h_{1} \in H^{\infty}$, we have $g \prec f$.

Corollary 3. Let $f$ and $g$ be analytic on $\mathbb{D}$. There exists an absolute constant $c_{0}$, with $0<c_{0}<1$, such that for any $c<c_{0}$, if

(i) $|g(z)| \leq|f(z)| \quad(c \leq|z|<1)$ and

(ii) $Z_{g} \backslash Z_{f} \subset\left\{z \in \mathbb{D}\left|c^{1 / 3}<\right| z \mid<1\right\}$,

then $\|g\|<\|f\|$.

Proof. If $\|f\|=\infty$, then there is nothing to prove. Thus we can assume that $f \in A_{2}$, which implies $g \in A_{2}$ by (i). Applying Theorem 3, we have $g \prec f$ and, in particular, $\|g\| \leq\|f\|$.

Notice that (i) and (ii) together imply that $Z_{g} \backslash Z_{f}$ is separated from $Z_{f} \backslash Z_{g}$ by the annulus $\left\{z \in \mathbb{D}|c \leq| z \mid \leq c^{1 / 3}\right\}$.

Proof of Theorem 1. Let $F=G / B$ where $B=\prod_{k=1}^{M} B_{a_{k}}$ and $\left\{a_{k}\right\}_{k=1}^{M}=Z_{G}$ $(M \leq \infty)$. Applying (4), we have for $\lambda, \gamma>0$

$$
\left|B_{a_{k}}\right|^{\lambda \gamma \alpha_{k}} \prec|z|^{\lambda \gamma}
$$

where $\alpha_{k}=\left(1+\left|a_{k}\right|\right) /\left(1-\left|a_{k}\right|\right)$. Since $F$ has no zeros in $\mathbb{D}$ and $\|F\|_{\infty} \leq 1$, we can define the analytic function $\widehat{F}=F^{2 \lambda \gamma / \log (1 /|F(0)|)}$ that satisfies $\|\widehat{F}\|_{\infty} \leq 1$ and $|\widehat{F}(0)|=e^{-2 \lambda \gamma}$. Thus, we can apply Proposition 2 to $\widehat{F}$ yielding

$$
\widehat{F} \prec|z|^{\lambda \gamma} \text {. }
$$

Now let $h \in H^{\infty}$. We have

$$
\begin{aligned}
& \int_{\mathbb{D}}|G h|^{2} d m=\int_{\mathbb{D}}\left|\prod_{k} B_{\alpha_{k}}(z)\right|^{2}|F(z) h(z)|^{2} d m \\
& \quad=\int_{\mathbb{D}} \prod_{k}\left(\left|B_{a_{k}}(z)\right|^{2 \lambda \gamma \alpha_{k}}\right)^{1 /\left(\lambda \gamma \alpha_{k}\right)}\left(|\widehat{F}(z)|^{2}\right)^{(\log 1 /|F(0)|) / 2 \lambda \gamma}|h(z)|^{2} d m,
\end{aligned}
$$

where we choose

$$
\lambda=\frac{1}{\gamma}\left[\sum_{k}\left(\frac{1}{\alpha_{k}}\right)+\frac{1}{2} \log \frac{1}{|F(0)|}\right] .
$$


For this $\lambda$, we can apply the arithmetic-geometric mean inequality to (10) to obtain

$$
\begin{aligned}
\int_{\mathbb{D}}|G h|^{2} d m \leq & \sum_{k} \frac{1}{\lambda \gamma \alpha_{k}} \int_{\mathbb{D}}\left(\left|B_{a_{k}}\right|^{\lambda \gamma \alpha_{k}}|h(z)|\right)^{2} d m \\
& +\frac{\log (1 /|F(0)|)}{2 \lambda \gamma} \int_{\mathbb{D}}|\widehat{F}(z) h(z)|^{2} d m .
\end{aligned}
$$

Applying (7) and (8), it follows that

$$
\begin{aligned}
\int_{\mathbb{D}}|G h|^{2} d m & \leq\left[\sum_{k}\left(\frac{1}{\lambda \gamma \alpha_{k}}\right)+\frac{\log (1 /|F(0)|)}{2 \lambda \gamma}\right] \int_{\mathbb{D}}|z|^{2 \lambda \gamma}|h(z)|^{2} d m \\
& =\int_{\mathbb{D}}|z|^{2 \lambda \gamma}|h(z)|^{2} d m,
\end{aligned}
$$

by the above choice of $\lambda$. We will show that $\lambda \geq 1$. Once this is done, it follows that

$$
\int_{\mathbb{D}}|G h|^{2} d m \leq \int_{\mathbb{D}}|z|^{2 \gamma}|h(z)|^{2} d m
$$

which is the desired result. To see that $\lambda \geq 1$, first observe that

$$
|F(0)| \prod_{k}\left|a_{k}\right|=|F(0) B(0)|=|G(0)| \leq c^{\gamma(1+c) /(1-c)},
$$

where the last inequality follows by hypothesis. This implies

$$
\frac{1+c}{1-c} \log \frac{1}{c} \leq \frac{1}{\gamma} \sum_{k}\left(\log \frac{1}{\left|a_{k}\right|}\right)+\frac{1}{\gamma} \log \frac{1}{|F(0)|} .
$$

It can be shown that the function $\phi(r)=\frac{1+r}{1-r} \log \frac{1}{r}$ is decreasing and $\phi(r)>2$ on $(0,1)$. We have, by assumption, that $\left|a_{k}\right|>c$ and thus, $\phi\left(\left|a_{k}\right|\right) \leq \phi(c)$ for all $k$. This yields

$$
\log \frac{1}{\left|a_{k}\right|} \leq\left(\frac{1-\left|a_{k}\right|}{1+\left|a_{k}\right|}\right) \frac{1+c}{1-c} \log \frac{1}{c}=\frac{1}{\alpha_{k}}\left(\frac{1+c}{1-c} \log \frac{1}{c}\right) .
$$

This, together with (12), implies

$$
\frac{1+c}{1-c} \log \frac{1}{c} \leq \frac{1}{\gamma}\left[\sum_{k}\left(\frac{1}{\alpha_{k}}\right) \frac{1+c}{1-c} \log \frac{1}{c}+\log \frac{1}{|F(0)|}\right]
$$

or

$$
\begin{aligned}
1 & \leq \frac{1}{\gamma}\left[\sum_{k}\left(\frac{1}{\alpha_{k}}\right)+\frac{2(1-c)}{(1+c) \log 1 / c} \cdot \frac{1}{2} \log \frac{1}{|F(0)|}\right] \\
& \leq \frac{1}{\gamma}\left[\sum_{k}\left(\frac{1}{\alpha_{k}}\right)+\frac{1}{2} \log \frac{1}{|F(0)|}\right]=\lambda,
\end{aligned}
$$

where the last inequality is a consequence of $\phi(c)>2$. This completes the proof of the theorem. 


\section{REFERENCES}

1. B. Korenblum, A maximum principle for the Bergman space, Publ. Mat. 35 (1991), 479-486.

2. _ Transformations of zero sets by contractive operators in the Bergman space, Bull. Sci. Math. (2) 114 (1990), 385-394.

3. B. Korenblum, R. O'Neil, K. Richards, and K. Zhu, Totally monotone functions with applications to the Bergman space, Trans. Amer. Math. Soc. (to appear).

Department of Mathematics, State University of New York, Albany, New York 12222

Department of Mathematics, Texas Tech University, Lubbock, Texas 79409

Current address, Kendall Richards: Department of Mathematics, Southwestern University, Georgetown, Texas 78627 